REFERENCIA: Marín-Liébana, P., Blasco, J.S., \& Botella, A.M. (2020). Hacia una comprensión de las identidades musicales de los estudiantes. Desde los primeros años hasta la adolescencia. ENSAYOS,

Revista de la Facultad de Educación de Albacete, 35(2). Enlace web: http://www.revista.uclm.es/index.php/ensayos - Consultada en fecha (dd-mm-aaaa)

\title{
HACIA UNA COMPRENSIÓN DE LAS IDENTIDADES MUSICALES DE LOS ESTUDIANTES. DESDE LOS PRIMEROS AÑOS HASTA LA ADOLESCENCIA
}

\section{TOWARDS UNDERSTANDING STUDENTS' MUSICAL IDENTITIES. FROM THE EARLY YEARS TO ADOLESCENCE}

\author{
Pablo Marín-Liébana \\ Pablo.Marin-Liebana@uv.es \\ José Salvador Blasco Magraner \\ J.salvador.blasco@uv.es \\ Ana María Botella Nicolás \\ Ana.Maria.Botella@uv.es \\ Facultat de Magisteri. Universitat de València (España)
}

Recibido: $16 / 04 / 2020$

Aceptado: 09/12/2020

\begin{abstract}
Resumen:
El interés por el reconocimiento de las identidades musicales de los estudiantes en el ámbito educativo ha crecido durante los últimos años. No obstante, se trata de un concepto ambiguo y de difícil acotación terminológica, lo que dificulta su comprensión e identificación. Aquí se realiza una revisión bibliográfica de 79 trabajos, estudiando su desarrollo teórico, su aplicación en la música y su evolución a lo largo de las etapas que comprenden desde los primeros años hasta la adolescencia. Así, se ha encontrado que las identidades musicales son complejos constructos interseccionales, discursivos y performativos que se elaboran en torno a las tensiones entre individuo-sociedad y juegan un importante papel en el desarrollo psicosocial de los estudiantes. Finalmente, se elabora una serie de propuestas de intervención educativa.
\end{abstract}

Palabras clave: Educación musical; educación primaria; educación secundaria; identidades; música.

\begin{abstract}
:
The interest in the recognition of the musical identities of students in the educational field has grown in recent years. However, it is an ambiguous concept and it is difficult to define, which makes it difficult to understand and identify. A literature review of 79 works was carried out, studying their theoretical development, their application to music and their evolution throughout the stages that comprise from the early years to adolescence. Thus, it has been found that musical identities are complex intersectional, discursive and performative constructs that are elaborated around the tensions between individual-society and play an important role in the psychosocial development of students. Finally, a series of proposals for educational intervention are presented.
\end{abstract}

Keywords: Music education; elementary education; secondary education; identities; music. 


\section{Introducción}

Desde que el concepto de identidad se hiciera popular como objeto de estudio en la década de 1950 (Gleason, 1983), este se ha convertido en uno de los términos más utilizados por las ciencias sociales y las humanidades, dando lugar en la actualidad a una gran pluralidad de aproximaciones y enfoques (Wetherell, 2010). De hecho, en el ámbito educativo ha crecido durante los últimos años el interés por reconocer e incorporar las identidades musicales de los estudiantes (Westerlund, Partti, y Karlslen, 2017). Sin embargo, el concepto de identidad es complejo y en ocasiones ambiguo, lo que dificulta entender con exactitud las identidades musicales y sus procesos de construcción a lo largo de las etapas educativas. Por tanto, el objetivo de este trabajo es el de elaborar una aproximación teórica a dicho concepto y establecer una serie de implicaciones educativas a partir de la misma. En última instancia, se trata de contribuir a que docentes e investigadores consigan un mayor nivel de comprensión y sugerir una serie de estrategias didácticas que tiendan hacia la construcción, reconocimiento e incorporación de las identidades musicales de los estudiantes en los espacios educativos.

\section{Metodología}

Para cumplir con el objetivo planteado, se ha llevado a cabo una revisión bibliográfica de tipo narrativo. En primer lugar, se realizó una búsqueda de información en las bases de datos Web of Science y Scopus, en la que se utilizó una sintaxis basada en los términos "musical identity" y education, localizando 43 artículos científicos. De estos, se descartaron los duplicados y aquellos que abordaban periodos evolutivos posteriores a la adolescencia. A partir de una primera lectura se identificaron los principales temas de interés y, mediante la técnica de snowball, se accedió a los trabajos que contaban con un mayor número de referencias y a los que trataban de forma específica la temática que aquí se aborda. En total, la muestra estudiada ascendió a 79 trabajos diferentes.

\section{Resultados}

Aquí se presentan los resultados obtenidos a partir de la revisión bibliográfica. Estos se organizan en torno a cuatro ejes: aclaración terminológica del concepto de identidad, principales corrientes teóricas, la identidad específicamente musical y su desarrollo hasta la adolescencia.

\subsection{Aclaración terminológica}

En torno al concepto de identidad giran habitualmente otros términos que, debido a su proximidad semántica, son en ocasiones utilizados indistintamente como sinónimos. Los dos más frecuentes son el yo y el autoconcepto o autoimagen. Por un lado, el yo se remonta a los inicios de la psicología, en los que James (1909) diferenciaba entre un sujeto pensante (I) que se pensaba como objeto $(m e)$. Actualmente, parece existir un consenso en torno a una naturaleza dual del yo según la cual este es tanto un sistema de acción dinámico, motivado cognitiva y emocionalmente, como un sistema de autoconstrucción interpersonal (Morf y Mischel, 2012).

Por otro lado, los autoconceptos o autoimágenes representan la manera en la que nos vemos en determinadas situaciones (Spychiger, 2017). Estos se integran y cobran sentido dentro de la identidad, combinándose en visiones más globales sobre nosotros mismos (Hargreaves y Lamont, 2017; Hargreaves, MacDonald, y Miell, 2016; Hargreaves, Miell, y MacDonald, 2002). 
Finalmente, el concepto de identidad consiste en "definirse a uno mismo en términos de valores, fortalezas, habilidades y objetivos, y en negociar un lugar para ese yo en el medio social" (Evans y McPherson, 2017, p. 213).

A modo de síntesis, Oyserman, Elmore y Smith (2012) proponen una precisión terminológica sobre los tres conceptos. En primer lugar, el yo es la sensación que implica la existencia de una capacidad reflexiva según la cual un sujeto piensa, es consciente de ello y se concibe a sí mismo como objeto de pensamiento. En segundo lugar, los autoconceptos son estructuras cognitivas que incluyen contenidos, actitudes y juicios de valor que dan sentido al mundo y a uno mismo dentro de él. Por último, la identidad es un marco de sentido mayor en el que se integran distintas partes del autoconcepto, tiene una dimensión personal y otra social, y puede ser colectiva.

\subsection{Corrientes teóricas en torno al concepto de identidad}

La identidad como objeto de estudio de las ciencias sociales contemporáneas puede rastrearse hasta las formulaciones realizadas por Williams James y George Herbert Mead entre finales del siglo XIX y principios del XX (Hammack, 2015; Hargreaves et al., 2002). Ambos coinciden en definirla como un proceso de percepción de unidad y coherencia, de continuidades y semejanzas sobre nuestra propia persona, que presenta una cierta independencia con respecto al paso del tiempo y al cambio de contexto (James, 1909; Mead, 1934). Sin embargo, aquellas características que diferencian a ambos autores se han convertido en una suerte de piedras fundacionales sobre las que se han desarrollado dos tradiciones académicas diferenciadas alrededor del concepto de identidad (Hammack, 2015). Por un lado, James otorga una mayor importancia al papel desempeñado por el mundo privado y el pensamiento interior en su desarrollo, dando lugar a las teorías de la crisis de identidad de Erikson (1956; 1968), de los estados de identidad de Marcia $(1966 ; 1980)$ y de los estilos de procesamiento de Berzonsky (1988; 2010). Por su parte, Mead concede una mayor centralidad al papel social que juega el mundo público y los significados simbólicos. Sobre él se han desarrollado las teorías del estigma de Goffman (1959; 1963), de los roles identitarios de McCall y Simmons (1978), de la identidad y el interaccionismo simbólico estructural de Stryker (1968; 2007), y de la identidad social de Tajfel y Turner (1974; 1986).

Siguiendo la estela de James, Erikson (1968) sostiene que el desarrollo de la identidad es un proceso que el ser humano experimenta a lo largo de toda la vida, pero que cobra un especial protagonismo en forma de crisis durante la etapa de la adolescencia. Según el propio Erickson, en este período el individuo trata de establecer puentes entre su identidad infantil y una adultez anticipada, así como entre su autoconcepto y la percepción que de él tienen los demás (Sneed, Schwartz, y Cross, 2006). El grado de éxito en la superación de esta crisis marcará una personalidad estable o un mantenimiento del estado de lucha por tratar de encontrar un lugar en el mundo.

En esta línea, Marcia $(1966 ; 1980)$ identifica cuatro estados en el desarrollo de la identidad que se definen por los valores que toman las dimensiones de exploración y compromiso (Dys, Schellenberg, y McLean, 2017). Así, aquellos que han explorado diferentes posibilidades y finalmente se han comprometido con una identidad tienen un estado de logro. Estos presentan una mayor madurez, pensamiento equilibrado, capacidad de toma de decisiones y fuertes relaciones sociales. Por otra parte, aquellos con un alto nivel de exploración, pero sin un compromiso efectivo, se encuentran en un estado de moratoria y presentan estrés e incertidumbre. En el caso de mostrar un compromiso identitario sin haber explorado distintas posibilidades, un individuo se encuentra en un estado de cerrazón. Las personas en esta situación están relacionadas con la resistencia al cambio y la impermeabilidad hacia las nuevas ideas. Finalmente, aquellos con bajos niveles de exploración y compromiso se sitúan en un estado difuso, caracterizado por la apatía, el desinterés y la incapacidad de acción. Este modelo 
ha dado lugar a numerosos estudios de carácter empírico en torno al concepto del desarrollo de la identidad en la adolescencia (Luyckx y Schwartz, 2008; Meeus, 2011). Más adelante, Berzonsky $(1988 ; 2010)$ añade al modelo de Marcia diferencias en el proceso por el cual los individuos toman decisiones personales y resuelven problemas.

Por otra parte, dentro de la tradición interaccionista iniciada por Mead, Goffman (1959; 1963) distingue entre la identidad personal y la identidad social. La primera la define como la diferenciación de uno mismo con respecto a los demás y la convergencia de los "hechos sociales de una única historia continua" (Goffman, 1963, p. 73). En cuanto a la identidad social, esta queda determinada por las categorizaciones y atributos que un contexto determinado dispone como posibilidades de inferencia sobre las características del otro en una interacción social. En la medida en que estas categorizaciones condicionan las posibilidades sociales de los individuos, estos realizan una autorepresentación biográfica para proyectar una imagen pública determinada sobre sí mismos. De un modo similar, McCall y Simmons (1978) definen los roles identitarios como las construcciones que hacen los individuos de sí mismos para poder ocupar una determinada posición social. En la misma línea, Stryker $(1968 ; 2007)$ acuña el concepto de interaccionismo simbólico estructural para resaltar la fuerza que tienen las estructuras sociales dentro de la mutua conformación entre individuo y sociedad.

Finalmente, Tajfel y Turner $(1974 ; 1986)$ definen la identidad social como aquellos aspectos de la autoimagen de un individuo que derivan de las categorías sociales, vinculadas a grupos, a las que cree pertenecer. Asumen que las personas queremos tener una buena identidad, que dichas categorías sociales reciben valores positivos o negativos de los grupos de pertenencia, y que dichos grupos se evalúan en comparación con aquellos a los que no se pertenece. A partir de esto, infieren que, para tener una positiva identidad social, los individuos deben percibir a sus grupos como positivamente diferenciados de los otros. En caso de no ser así, tratarán de cambiar de grupo o hacer que este tenga un mayor grado de diferenciación positiva con el resto.

Dentro de estas dos grandes tradiciones, los investigadores han abordado el concepto de identidad como una característica del sujeto a partir de categorías más o menos estables y autónomas como raza, clase social, género, religión, nacionalidad, sexualidad o discapacidad. Sin embargo, a partir de la década de 1980, estos enfoques entraron en crisis como consecuencia de los desarrollos teóricos del posestructuralismo, el posmodernismo, la deconstrucción, los estudios sobre performatividad y la teoría queer, así como el giro discursivo, las teorías poscolonialistas y el relativismo cultural (Wetherell, 2010).

Por un lado, la identidad dejó de considerarse como una esencia del sujeto situada en la esfera privada de la cognición, la emoción y la experiencia, para empezar a entenderse como un discurso en construcción situado en la esfera pública de las interacciones y otros sistemas semióticos de generación de significado (Benwell y Stokoe, 2010). El referente principal de este giro discursivo es Foucault (1976; 1978), según el cual la identidad se inscribe en los discursos dominantes y en las prácticas sociales e institucionales, de forma que el sujeto se encuentra estructurado por estos y perpetúa las relaciones de poder existentes. Una extensión de esta aproximación es la teoría de la performatividad (Butler, 2001; Chinn, 2010), que sostiene que la identidad se materializa en las acciones y decisiones de los individuos, las cuales no necesariamente tienen que estar vinculadas a una abstracción discursiva. Otras teorías en la línea del giro discursivo son los análisis críticos del discurso (Reisigl, 2013), la teoría posicionada (Harré, 2012), los enfoques narrativos (Schechtman, 2011) y la psicología discursiva crítica (Potter y Wetherell, 1987).

Por el otro lado, los trabajos realizados en el marco de la globalización, los movimientos migratorios y las redes sociales introdujeron una nueva perspectiva que sostenía que la identidad era algo más fluido, rico y diverso de lo que se había pensado previamente (Wetherell, 2010). Dentro de este nuevo enfoque se desarrolló la teoría de la interseccionalidad (Crenshaw, 
2016), según la cual los individuos están simultáneamente posicionados en diversas categorías (e.g., raza, clase social, género, religión, nacionalidad, etc.) por lo que no es posible comprender la identidad analizando solo una de ellas, sino que estas deben entenderse en el contexto de sus relaciones con las demás (Chinn, 2010).

\subsection{Concepto de identidad musical}

Como se ha visto, la identidad es un concepto complejo que ha sido abordado desde diferentes perspectivas y análisis, resistiéndose a una definición monolítica y ampliamente aceptada. Sin embargo, en el caso específico de la identidad musical, Green (2011) formula una definición integradora que incluye preferencias, habilidades, valores, prácticas y conocimientos musicales, así como los contextos en los que han sido adquiridos y desarrollados, todo ello en una doble dimensión que considera tanto al individuo como los sistemas sociales de los que forma parte, desde la familia hasta el estado-nación.

Por otra parte, Hargreaves, MacDonald y Miell (2002; 2009; 2017) realizan una interesante diferenciación conceptual dentro de las identidades musicales: las identidades en la música (IEM) y la música en las identidades (MEI). Las primeras están formadas por roles social y culturalmente establecidos dentro de la música y en él se encuentran categorías genéricas relacionadas con las actividades musicales, así como categorías más específicas vinculadas a instrumentos o géneros musicales. Por ejemplo, los roles de músico, compositor, intérprete, docente, crítico o consumidor/público/fan son ejemplos de identidades genéricas en la música, mientras que conceptos como viento madera, cuerda frotada o percusión son ejemplos de identidades específicas vinculadas a instrumentos.

Asimismo, etiquetas como pop, rock, jazz o música clásica están relacionadas con los géneros musicales. Tanto las categorías genéricas como las específicas intersectan en cada individuo, quien puede adoptar una combinación de las mismas. Estas categorías, que gozan de un carácter universal, son reforzadas por instituciones como escuelas y conservatorios (Hargreaves et al., 2017). Por otra parte, el segundo grupo está constituido por las formas en que la música desarrolla diferentes aspectos de nuestra identidad general, como pueden ser las identidades vinculadas con el género, la edad, la nacionalidad, la diversidad funcional o la propia personalidad.

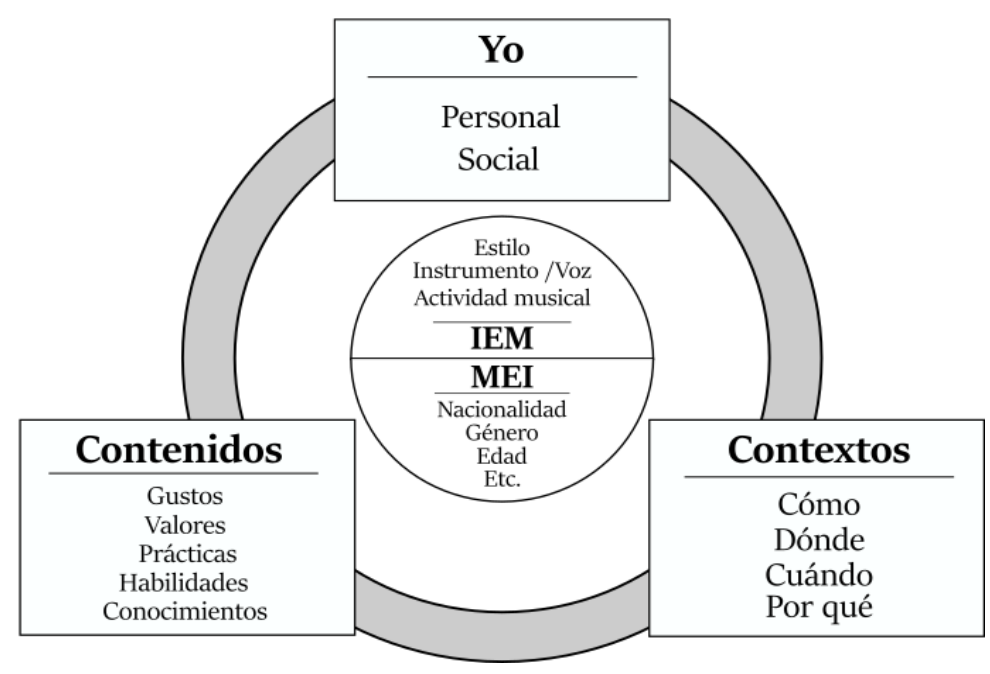

Figura 1. Concepto de identidad musical (elaboración propia). 
Tanto la aproximación de Green (2011, p. 1) como la diferenciación de Hargreaves, MacDonald y Miell (2017, 2002; MacDonald et al., 2009) pueden ser integradas en un mismo esquema conceptual que operativiza el uso de la identidad musical como objeto de estudio y categoría de análisis (figura 1). En él, las IEM y las MEI quedan configuradas por la relación que se produce entre las dimensiones del yo, los contenidos identitarios y los contextos de producción de los mismos.

\subsection{Desarrollo de la identidad musical hasta la adolescencia}

En tanto que proceso de subjetivización de la experiencia del individuo, la identidad no es un elemento estable a lo largo de la vida, sino que su desarrollo está íntimamente ligado al de la propia persona. En este sentido, apoyándose en la teoría ecológica de Bronfebrenner (1979), Lamont $(2002,2017)$ propone un esquema del desarrollo de la identidad que transita desde una mayor predominancia de la dimensión personal hacia un mayor protagonismo del componente social. Así, sostiene que el desarrollo de la identidad en los primeros años se caracteriza por la dimensión física y observable, es inestable en el tiempo y suele ser global y poco preciso. Más adelante, presenta una mayor continuidad temporal y es específico de cada habilidad. Entre los 6 y los 13 años, como consecuencia de la comparación con el grupo de iguales, la predominancia de lo físico disminuye en favor de lo psicológico y lo social, siendo estos factores claves durante la adolescencia. Aplicando esto al terreno musical, la autora señala que las identidades se construyen inicialmente a través de experiencias externas observables que cobran especial fuerza en el seno de un grupo social. Durante la etapa aproximada entre 6 y 12 años, el grupo de iguales representa el agente de mayor influencia, mientras que en la adolescencia las identidades musicales están relacionadas con las emociones y las actitudes. Además, en todas las etapas, los contextos de desarrollo ejercen un marcado condicionamiento.

Profundizando en cada una de ellas, durante los primeros años de vida, la identidad musical se desarrolla a través de las interacciones con los cuidadores en forma de juegos, historias y canciones (Hargreaves et al., 2017). En esta fase, Trevarthen (2002) sostiene que la música juega un importante rol en la construcción de la memoria y el desarrollo de la identidad como consecuencia de su carga emocional. Así, a los pocos meses, un bebé puede hacer música y se puede observar en él una preferencia hacia determinados patrones que toma del entorno y que, por tanto, suponen una primera identidad social como miembro de un grupo (Trevarthen y Malloch, 2017).

Durante la infancia, los individuos desarrollan su identidad musical participando en la música de su marco cultural y relacionándola con su propio bagaje (Hargreaves et al., 2017). La escuela juega un importante papel en el desarrollo de las identidades musicales. Por un lado, los escolares aprenden los valores y habilidades que constituyen la multifacética identidad de una persona vinculada a la música: tocar un instrumento y/o cantar, actuar frente a otros, enseñar música, leer notación musical, dirigir una agrupación, hablar sobre la propia música y respetar la diversidad de gustos (Lamont, 2017). Sin embargo, algunas investigaciones de finales de la década de 1990 demostraron que solo un $7 \%$ de los estudiantes de secundaria elegían la asignatura optativa de música (Bray, 2000), y que quienes lo hacían solían ser estudiantes que recibían formación musical extraescolar. Esto sugiere que la escuela primaria implementa un sistema vinculado con la música profesional y no tanto con la música desde una perspectiva inclusiva, lo que influye de manera negativa en las identidades que los estudiantes construyen de sí mismos, quienes rechazan la asignatura (Lamont, 2002). Esta idea se refuerza con algunos trabajos que señalan al declive que experimenta la asignatura de educación musical en la etapa de secundaria (Harland et al., 2000; Ross, 1995).

Otros autores (Lamont, 1998, 2002; Lamont y Tarrant, 2001) han estudiado también la influencia de determinados factores en el desarrollo de la identidad musical durante la infancia. Así, concluyen que esta es más positiva en los individuos de menor edad, en aquellos que provienen 
de familias con vinculación musical, en los que participan en escuelas con metodologías inclusivas y en el género femenino. Por el contrario, una identidad musical negativa se asocia con un bajo nivel socioeconómico, con metodologías elitistas o profesionalizantes, con la no participación en actividades extraescolares de música en contextos en los que esta actividad es frecuente, con la etapa de secundaria y con el género masculino.

En la adolescencia, escuchar música es una de las actividades favoritas de los jóvenes (J. D. Brown y Bobkowski, 2011; Fitzgerald, Joseph, Hayes, y O'regan, 1995) y esta contribuye determinantemente al desarrollo de su identidad (Arnett, 1995; Evans y McPherson, 2017; Hargreaves, MacDonald, y Miell, 2012; McPherson, Davidson, y Faulkner, 2012; Miranda, 2013; Shaw, Kleiber, y Caldwell, 1995; Thomas, 2016). En esta fase de la vida, las experiencias que tienen lugar fuera de las instituciones escolares son las que ejercen una mayor influencia en su conformación (North, Hargreaves, y O’Neill, 2000).

El atractivo de la música para los adolescentes, particularmente la música popular urbana, parece estar relacionado con determinadas necesidades evolutivas (Tarrant, North, y Hargreaves, 2002). Por ejemplo, las letras de las canciones pueden servir como apoyo en la construcción de sus identidades sexuales y sus roles de género, en la comprensión de la transición entre la infancia y la vida adulta o en la identificación con distintas formas de rebelión (Larson y Kubey, 1983). De hecho, este repertorio es especialmente importante en la construcción de sus identidades y es el medio a través del cual se sitúan a favor o en contra de los discursos que les rodean (Westerlund et al., 2017). Así, Künzler (2011) estudia cómo el rap sirve para que los jóvenes sudafricanos que crecieron en el final del apartheid desarrollen una identidad urbana y moderna a la vez global y local. Del mismo modo, Kelly, Pomerantz y Currie (2005) apuntan que estilos como el rock alternativo, el punk o el metal contribuyen en la construcción de la identidad de chicas skaters y feministas en Vancouver. No obstante, cada persona puede elaborar o reforzar identidades opuestas a partir de los mismos contenidos, tal y como apuntan McKinlay y McVittie (2017). Por otra parte, algunos estudios relacionan el consumo de determinada música con la realización de determinadas actividades y el desarrollo de algunos tipos de actitudes. Así, Pardun, L'Engle y Brown (2005) establecen una relación entre el consumo de música y la cantidad de actividad sexual, mientras que Ward, Hansbrough y Walker (2005) sostienen que aquellos individuos que consumen videoclips en mayor cantidad presentan unas actitudes más tradicionales en relación con los roles de género y las relaciones sexuales.

Las funciones de identidad que cumple la música para los adolescentes son tanto personales como sociales (Tarrant et al., 2002). Por un lado, estos la utilizan para regular su estado de ánimo (Behne, 1997; North et al., 2000; Roe, 1985; Saarikallio y Erkkilä, 2007), enfrentarse o sobrellevar situaciones difíciles (Kurdek, 1987) o compensar un bajo autoconcepto de su apariencia física (Kistler, Rodgers, Power, Austin, y Hill, 2010). Además, la escucha privada es un espacio en el que las imágenes y las emociones de la música ayudan explorar identidades alternativas (Larson, 1995). Por el otro, tanto el grupo de iguales (Johnstone y Katz, 1957) como la deseabilidad normativa dentro de los diferentes estilos (North y Hargreaves, 1999), parecen condicionar las preferencias de los adolescentes, quienes utilizan la música para proyectar una imagen sobre los demás y satisfacer las expectativas que puedan tener sobre ellos mismos (North et al., 2000; Tarrant y North, 2000). Así, la música ejerce la función de definir las características de un grupo de pertenencia y sus diferencias con el resto (Hargreaves et al., 2017). En este sentido, Finnäs (1989) sostiene que los adolescentes expresan diferentes gustos musicales en público y en privado con el objetivo de adecuarse a las características de sus grupos de iguales. Del mismo modo, Brown y O'leary (1971) sugieren que la MPU puede servir a los que no destacan académicamente para tener una buena aceptación en dichos grupos. 
A modo de síntesis, la tabla 1 muestra cómo la conformación de la identidad musical transita por tres etapas evolutiva. Las características principales se han agrupado en torno a sus contextos de construcción, los contenidos y funciones que desempeña y la tendencia general que sigue desde los primeros años hasta el inicio de la edad adulta. Como se puede observar, la segunda infancia y la adolescencia se solapan, lo que es debido a los diferentes ritmos evolutivos que tienen lugar en esta transición.

\begin{tabular}{|c|c|c|c|}
\hline & $\begin{array}{l}\text { Infancia temprana } \\
\qquad(0-6)\end{array}$ & $\begin{array}{l}\text { Segunda infancia } \\
(6-12)\end{array}$ & Adolescencia (10-21) \\
\hline \multirow{2}{*}{$\begin{array}{l}\text { Contextos de } \\
\text { construcción }\end{array}$} & $\begin{array}{l}\text { Espacio físico } \\
\text { observable }\end{array}$ & Grupo de iguales & Grupo de iguales \\
\hline & $\begin{array}{l}\text { Interacción con } \\
\text { cuidadores }\end{array}$ & Contexto escolar & Contexto extraescolar \\
\hline \multirow{3}{*}{$\begin{array}{l}\text { Contenido y } \\
\text { función }\end{array}$} & & \multirow{3}{*}{$\begin{array}{l}\text { Experimentación } \\
\text { sobre los roles } \\
\text { musicales }\end{array}$} & Preferencia por la escucha \\
\hline & $\begin{array}{l}\text { Construcción de la } \\
\text { memoria }\end{array}$ & & $\begin{array}{c}\text { Contribuye a la construcción de la } \\
\text { identidad sexual, los roles de género y } \\
\text { la ideología }\end{array}$ \\
\hline & $\begin{array}{l}\text { Preferencia por } \\
\text { patrones sonoros }\end{array}$ & & $\begin{array}{l}\text { Es utilizada para regular el estado de } \\
\text { ánimo, enfrentar situaciones difíciles, } \\
\text { mejorar el autoconcepto y definir el } \\
\text { grupo de pertenencia }\end{array}$ \\
\hline $\begin{array}{l}\text { Tendencia } \\
\text { general }\end{array}$ & $\begin{array}{c}\text { Más personal, } \\
\text { discontinua y global }\end{array}$ & \multicolumn{2}{|c|}{ Más social, estable y específica } \\
\hline
\end{tabular}

Tabla 1. Evolución de la identidad musical (elaboración propia).

\section{Conclusiones e implicaciones educativas}

Este trabajo ha aportado un extenso marco conceptual a partir del cual comprender las identidades musicales de los estudiantes, sus procesos de construcción y las funciones que estas pueden desempeñar. Así, se ha visto que la identidad es un proceso de percepción de unidad, coherencia, continuidad y semejanza sobre la propia persona que constituye un marco de sentido en una doble dimensión individual y social en el que se integra el autoconcepto. Si bien las teorías clásicas se decantaban más por la dimensión psicológica, representando un importante papel la crisis de la adolescencia, o por la sociológica, en torno a los roles sociales, la pertenencia a colectivos y la influencia del grupo de iguales, a partir de la década de 1980 se han desarrollado nuevas teorías que han añadido otros elementos de análisis. En este sentido, la identidad ya no se concibe como una característica del sujeto, sino como un discurso en construcción con el que se genera una narrativa orgánica sobre la propia biografía, una acción performativa mediante la cual se toman decisiones contingentes sin la necesidad de una abstracción discursiva, o un elemento interseccional que resulta de la combinación de las diferentes categorías y sistemas sociales a los que pertenece el individuo.

En cuanto a la identidad musical, esta se construye a partir del conjunto de las experiencias musical personales y de pertenencia a colectivos; de sus gustos, valores, prácticas, habilidades y conocimiento; y de los contextos en los que todo lo anterior se desarrolla o tiene lugar. 
Además, se ha diferenciado entre identidades en la música (IEM) y música en las identidades (MEI), estando las primeras formadas por roles musicales socialmente establecidos como el de intérprete, compositor, docente, crítico o consumidor, entre otros, y las segundas por la forma en que la música perfila dimensiones identitarias no específicamente musicales como el género, la raza, la edad o la nacionalidad.

Como se ha visto, la construcción de la identidad musical desde los primeros años de vida hasta la adolescencia experimenta diferentes fases o etapas, las cuales pueden sintetizarse como una transición desde una dimensión más personal, física y observable hasta una más social, semántica y discursiva. Además, si bien al principio el marco social se sitúa en los cuidadores, tanto en el ámbito familiar como en el escolar, al llegar a la adolescencia este se define por el grupo de iguales y los contextos que se encuentran fuera de las instituciones educativas. En esa fase, los estudiantes se decantan principalmente por la música popular urbana y esta ejerce una importante función social en la construcción de sus identidades sexuales y roles de género, en la transición a la vida adulta, en la relación entro lo global y lo local, en la regulación del estado de ánimo y la compensación de un bajo autoconcepto, o en la exploración de identidades alternativas.

Por tanto, dado que las identidades musicales de los estudiantes son un elemento psicosocial de gran importancia en el desarrollo del individuo, los diferentes sistemas educativos deberían establecer estrategias para su desarrollo, reconocimiento e incorporación curricular, proponiendo procesos educativos de acompañamiento en la formación de las mismas. A la luz de los resultados expuestos, se podrían diferenciar tres etapas evolutivas, cada una con características identitarias diferentes y, por tanto, con la necesidad de intervenciones acordes a las mismas: la infancia temprana, la segunda infancia y la adolescencia. Además, con cierta flexibilidad en sus límites inferiores y superiores, estas se corresponden, respectivamente, con las etapas educativas de infantil, primaria y secundaria.

En este sentido, la primera fase debería abordarse con un doble objetivo: la exploración sonora del entorno social y natural, y el establecimiento de lazos afectivos positivos con respecto a la música. En el primero, quedarían incluidas propuestas pedagógicas como las planteadas por Akoschky (2005), quien sugiere comenzar con el trabajo del timbre a partir de los diferentes materiales (metal, madera, papel, plástico, etc.) y los posibles modos de acción (chocar, raspar, frotar, soplar, etc.). Se trata de manipular los objetos próximos para experimentar con sus posibilidades sonoras, pudiendo construir a partir de ellos lo que la autora denomina "cotidiáfonos". Igualmente, sería conveniente iniciar procesos de escucha a través de juegos motóricos y de expresión corporal, así como comenzar con la elaboración de un pequeño cancionero personal. En cuanto al establecimiento de vínculos emocionales positivos con respecto a la música, será necesario respetar los ritmos de aprendizaje y necesidades del alumnado, tratando de ampliarlos de forma suave y respetuosa con sus intereses. En este sentido, merece la pena señalar, por un lado, que no hay estudios que demuestren que el entrenamiento musical en edades preescolares sea necesario para llegar a un alto nivel musical en la adultez y, por el otro, que el desarrollo musical se ve favorecido por un entorno que apoye las necesidades de los niños y niñas, y que fomente la expresividad espontánea, siendo el apoyo familiar y la personalidad de los docentes algunos de los factores determinantes (Hodges, 2006; Trehub, 2006). En definitiva, en esta etapa se trata de construir un registro sonoro que contenga la mayor diversidad posible de referentes musicales y que estos estén vinculados con emociones y relaciones positivas.

Con respecto a la segunda infancia, sería interesante la elaboración de propuestas que ofrezcan una gran variedad y diversidad de experiencias musicales. Estas abarcan la experimentación tanto de los diferentes roles vinculados a la música, como de los diversos instrumentos y géneros o estilos. Además, estas experiencias deberán estar adaptadas a las características del alumnado 
y ser inclusivas para que la identidad sea construida de forma positiva. Es decir, enfoques basados en la notación escrita, la teoría musical, la precisión técnica y el repertorio académico podrían ser excesivamente difíciles, favorecer al alumnado con formación musical extraescolar y generar identidades musicales negativas y de rechazo en los estudiantes sin esas destrezas (Kertz-Welzel, 2013). En su lugar, los enfoques activos y prácticos, basados en la audición activa, el movimiento, la exploración instrumental y vocal, la creatividad y el juego, podrían contribuir a la construcción de identidades musicales más positivas durante la infancia. Estas, a su vez, conseguirían en etapas posteriores un mayor compromiso y voluntad de continuidad con la formación musical. Por tanto, el objetivo principal de esta fase es el de continuar con el vínculo afectivo iniciado durante la primera infancia y ampliar la cantidad de experiencias identitarias, de forma que se vaya consolidando una primera biografía musical que incluya tanto habilidades y conocimientos, como un registro sonoro en creciente ampliación.

Por último, en la adolescencia, si bien es necesario continuar con propuestas que enriquezcan las identidades de los estudiantes, ofreciéndoles modelos que amplíen su conocimiento y experiencias, estas deben ser complementadas con procesos de reconocimiento curricular. Es decir, en un momento en el que, siguiendo el modelo de Marcia $(1966,1980)$, los individuos deben explorar y adquirir una variedad de compromisos identitarios para alcanzar un estado de logro, el sistema educativo podría ser un espacio para ofrecerles esta posibilidad, y acompañarlos en el proceso, reconociendo e incorporando en el aula de música las identidades musicales transitorias por las que van pasando.

En este sentido, una posibilidad de reconocimiento e incorporación de las identidades musicales de los estudiantes durante la etapa de la adolescencia es la utilización de sus preferencias musicales como parte del repertorio escolar. Para ello, se podrían diseñar y programar actividades que se correspondan con las características contemporáneas atribuidas a la identidad, esto es, la interseccionalidad, la discursividad y la performatividad. Con respecto a la primera, por un lado, dado el contexto de diversidad cultural que existe en la mayoría de centros educativos, sería interesante profundizar en las diferentes biografías que interactúan en el aula para poder conocer las experiencias musicales que cada uno lleva a la escuela y hacer conscientes y explícitos los procesos de identificación musical, mostrando así una gran variedad de formas de relacionarse con la música. A partir de dicho conocimiento, se podrían utilizar metodologías participativas y democráticas a través de las que negociar los contenidos curriculares, de forma que las preferencias mayoritarias pasaran a forman parte del currículum impartido.

Con respecto a la segunda, habilitar espacios de investigación, exposición y reflexión sobre las preferencias musicales de los estudiantes podría contribuir a la construcción de la dimensión discursiva de sus identidades. De esta forma, sería interesante que buscasen información sobre sus artistas y canciones preferidos, que la compartiesen con el resto de la clase y que debatieran sobre algunas de las relaciones entre dicha música y los problemas sociales propios de su contemporaneidad. Esto les podría aportar herramientas de análisis crítico de su entorno y estrategias para la acción sobre el mismo. En cuanto a la tercera, la propia práctica musical a través de la voz, los instrumentos y el movimiento, contribuiría a la exploración identitaria performativa, sin perjuicio de que cada una de las propuestas nombradas anteriormente son en sí mismas también acciones realizadas.

Por otra parte, teniendo en cuenta que durante la adolescencia es especialmente relevante la música popular urbana, sería conveniente introducir estrategias de aprendizaje propias de este estilo, tal y como sugieren diversos autores bajo el nombre de aprendizaje informal (Chua y Ho, 2017; Davis, 2013; Green, 2004, 2006; Snell, 2009; Wright, 2008). De forma sintética, se trataría de utilizar piezas elegidas por los estudiantes, desarrollar la creatividad y el pensamiento crítico, fomentar un aprendizaje más horizontal a través de equipos colaborativos, acceder a la música 
a través de la escucha, y utilizar tecnologías digitales y electrónicas relacionadas con el estilo musical.

A modo de síntesis, la tabla 2 recoge las propuestas educativas planteadas para cada una de las etapas abordadas. Hay que tener en cuenta que las fronteras entre dichas etapas no representan un corte, sino un espacio de transición que variará entre los individuos debido a diversos factores biológicos, psicológicos y sociales. Por este motivo, algunas de las propuestas relativas a la infancia temprana podrían conservar su sentido durante los primeros años de la segunda infancia, del mismo modo que las correspondientes a la adolescencia podrían anticiparse durante los últimos cursos de la educación primaria.

\begin{tabular}{ccc}
\hline Infancia temprana (0-6) & Segunda infancia (6-12) & Adolescencia (10-21) \\
\hline $\begin{array}{c}\text { Exploración sonora del medio } \\
\text { social y natural }\end{array}$ & $\begin{array}{c}\text { Enfoques prácticos y activos: } \\
\text { audición activa, movimiento y } \\
\text { creatividad }\end{array}$ & $\begin{array}{c}\text { Reconocimiento y participación } \\
\text { curricular }\end{array}$ \\
$\begin{array}{c}\text { Ruegos expresivos } \\
\text { Relación afectiva a través del } \\
\text { apoyo social y el respeto a los } \\
\text { intereses propios }\end{array}$ & $\begin{array}{c}\text { Exploración de los diferentes } \\
\text { roles, géneros e instrumentos }\end{array}$ & $\begin{array}{c}\text { Desarrollo de las dimensiones } \\
\text { intersecional, discursiva y } \\
\text { performativa }\end{array}$ \\
\hline
\end{tabular}

Tabla 2. Propuestas educativas (elaboración propia).

Finalmente, desarrollar y reconocer las identidades de los estudiantes implica la incorporación de una visión de las manifestaciones musicales en tanto que elementos culturales que contienen significados y son utilizados por los individuos para definirse a sí mismos y proyectarse hacia el espacio social, en lugar de pensarlas como objetos autónomos y cerrados a los que acceder mediante una correcta formación. Este cambio de perspectiva constituye un primer paso hacia un modelo escolar preparado para participar conscientemente en el proceso de construcción identitaria de sus estudiantes.

\section{Referencias bibliográficas}

Akoschky, J. (2005). Los "cotidiáfonos" en educación infantil. Eufonía, 33, 1-10. https://www.grao.com/es/producto/los-cotidiafonos-en-la-educacion-infantileu03312637

Arnett, J. (1995). Adolescents' uses of media for self-socialization. Journal of Youth and Adolescence, 24(5), 519-533.

Behne, K. (1997). The development of "Musikerleben» in adolescence: how and why young people listen to music. En Perception and Cognition of Music (pp. 143-159). Hove: Psychology Press.

Benwell, B. y Stokoe, E. (2010). Analysing Identity in Interaction: Constrasting Discourse, Genealogial, Narrative and Conversation Analysis. En M. Wetherell y C. Mohanty (Eds.), The SAGE Handbook of Identities (pp. 82-103). Londres: SAGE.

Berzonsky, M. D. (1988). Self-theorists, identity status and social cognition. En D. Lapsley y P. F 
(Eds.), Self, ego and identity: Integrative approaches (pp. 243-262). New York: SpringerVerlag.

Berzonsky, M. D. (2010). Cognitive Processes and Identity Formation : The Mediating Role of Identity Processing Style. Psychologia rozwojowa, 15(4), 13-27.

Bray, D. (2000). An examination of GCSE music uptake rates. British Journal of Music Education, 17(1), 79-89. https://doi.org/10.1017/S0265051700000176

Bronfenbrenner, U. (1979). The Ecology of Humn Development: Experiments by Nature and Design. Cambridge: Harvard University Press.

Brown, J. D. y Bobkowski, P. S. (2011). Older and newer media: Patterns of use and effects on adolescents' health and well-being. Journal of Research on Adolescence, 21(1), 95-113. https://doi.org/10.1111/j.1532-7795.2010.00717.x

Brown, R. y O'leary, M. (1971). Pop Music in an English Secondary School System. American Behavioral Scientist, 14, 400-413.

Butler, J. (2001). El género en disputa: el feminismo y la subversión de la identidad. México: Paidós.

Chinn, S. E. (2010). Performative Identities: From Identity Politics to Queer Theory. En M. Wetherell y C. T. Mohanty (Eds.), The SAGE Handbook of Identities (pp. 104-124). Londres: SAGE Publications.

Chua, S. L. y Ho, H. P. (2017). Towards 21st-century music teaching-learning. Reflections on student-centric pedagogic practices involving popular music in Singapore. En G. D. Smith, Z. Moir, M. Brennan, S. Rambarran y P. Kirkman (Eds.), The Routledge Research Companion to Popular Music Education, (pp. 1-13). Routledge. https://doi.org/10.4324/9781315613444

Crenshaw, K. (2016). On interseccionality: Essential writings. Nueva York: New York New Press.

Davis, S. G. (2013). Informal learning processes in an elementary music classroom. Bulletin of the Council for Research in Music Education, 198, 23-50. https://doi.org/10.5406/bulcouresmusedu.198.0023

Dys, S. P., Schellenberg, G. y McLean, K. C. (2017). Musical Identities, Music Preferences and Individual Differences. En R. MacDonald, D. J. Hargreaves, y D. Miell (Eds.), Handbook of musical identities (pp. 247-266). New York: Oxford University Press.

Erikson, E. H. (1956). The problem of ego identity. Journal of the American Psychoanalytic Association, 4, 56-121.

Erikson, E. H. (1968). Identity. Youth and Crisis. New York: Norton and Company.

Evans, P. y McPherson, G. (2017). Processes of Musical Identity Consolidation during Adolescence. En R. MacDonald, D. J. Hargreaves, y D. Miell (Eds.), Handbook of musical identities (pp. 213-231). New York: Oxford University Press.

Finnäs, L. (1989). A comparison between young people's privately and publicly expressed musical preferences. Psychology of Music, 17, 132-145.

Fitzgerald, M., Joseph, A. P., Hayes, M. y O'regan, M. (1995). Leisure activities of adolescent schoolchildren. Journal of Adolescence. https://doi.org/10.1006/jado.1995.1024

Foucault, M. (1976). Vigilar y castigar: nacimiento de la prisión. Madrid: SIGLO XXI de España.

Foucault, M. (1978). La arqueología del saber. México: Siglo XXI. 
Gleason, P. (1983). Identifying Identity : A Semantic History. The Journal of American History, 69(4), 910-931.

Goffman, E. (1959). The presentation of self in everyday life. New York: Doubleday.

Goffman, E. (1963). Stigma; notes on the management of spoiled identity. New Jersey: PrenticeHall.

Green, L. (2004). What Can Music Educators Learn from Popular Musicians? En C. X. Rodríguez (Ed.), Bridging the Gap. Popular Music and Music Education (pp. 225-247). MENC: The National Association for Music Education.

Green, L. (2006). Popular music education in and for itself, and for "other» music: Current research in the classroom. International Journal of Music Education, 24(2), 101-118. https://doi.org/10.1177/0255761406065471

Green, L. (2011). The globalization and Localization of Learning, teaching, and Musical Identity. En L. Green (Ed.), Learning, Teaching, and Musical Identity: Voices across cultures (pp. 119). Bloomington: Indiana University Press.

Hammack, P. L. (2015). Theoretical Foundations of Identity. En K. C. McLean y M. Syed (Eds.), The Oxford Handbook of Identity Development (pp. 1-34). Oxford: Oxford University Press. https://doi.org/10.1093/oxfordhb/9780199936564.013.027

Hargreaves, D. J. y Lamont, A. (2017). The Psychology of Musical Development. Cambridge: Cambridge University Press.

Hargreaves, D. J., MacDonald, R. y Miell, D. (2012). Musical Identities Mediate Musical Development. En G. McPherson y G. Welch (Eds.), The Oxford Handbook of Music Education (pp. 125-142). New York: Oxford University Press.

Hargreaves, D. J., MacDonald, R. y Miell, D. (2016). Musical Identities. En S. Hallam, I. Cross, y M. Thaut (Eds.), The Oxford Handbook of Music Psychology (pp. 1-21). New York: Oxford University Press. https://doi.org/10.1093/oxfordhb/9780199730810.013.0008

Hargreaves, D. J., MacDonald, R. y Miell, D. (2017). The changing identity of musical identities. En R. MacDonald, D. J. Hargreaves, y D. Miell (Eds.), Handbook of musical identities (pp. 3-23). New York: Oxford University Press.

Hargreaves, D. J., Miell, D. y MacDonald, R. (2002). What are musical identities, and why are they important? En R. MacDonald, D. J. Hargreaves, y D. Miell (Eds.), Musical Identities (pp. 1-20). Oxford University Press.

Harland, J., Kinder, K., Lord, P., Scott, A., Schagen, I. y J, H. (2000). Arts in Education in Secondary Schools: Effects and Effectiveness. Slough: National Foundation for Educational Research.

Harré, R. (2012). Positioning Theory: Moral Dimensions of Social-Cultural Psychology. En J. Valsiner (Ed.), The Oxford Handbook of Culture and Psychology (pp. 1-28). Nueva York: Oxford University Press.

Hodges, D. A. (2006). The musical brain. En G. McPherson (Ed.), The Child as Musician. A Handbook of Musical Development, (pp. 51-68).

James, W. (1909). Principios de psicología. Madrid: Daniel Jorro.

Johnstone, J. y Katz, E. (1957). Youth and Popular Music: A Study in the Sociology of Taste. American Journal of Sociology, 62(6), 670-672. https://doi.org/10.1086/222104

Kelly, D. M., Pomerantz, S. y Currie, D. (2005). Skater girlhood and emphasized femininity: «you can't land an ollie properly in heels». Gender and Education, 17(3), 229-248. 


\section{https://doi.org/10.1080/09540250500145163}

Kertz-Welzel, A. (2013). Children's and adolescents' musical needs and music education in Germany. En P. S. Campbell y Trevor Wiggins (Eds.), Children's Musical Cultures (pp. 371386). Oxford University Press. https://doi.org/10.1093/oxfordhb/9780199737635.001.0001

Kistler, M., Rodgers, K. B., Power, T., Austin, E. W. y Hill, L. G. (2010). Adolescents and music media: Toward an involvement-mediational model of consumption and self-concept. Journal of Research on Adolescence, 20(3), 616-630. https://doi.org/10.1111/j.15327795.2010.00651.x

Künzler, D. (2011). South African rap music, counter discourses, identity, and commodification beyond the prophets of Da City. Journal of Southern African Studies, 37(1), 27-43. https://doi.org/10.1080/03057070.2011.552540

Kurdek, L. (1987). Gender differences in the psychological symptomatology and coping strategies of young adolescents. Journal of Early Adolescence, 7(4), 395-410.

Lamont, A. (1998). Music, education, and the development of pitch perception: the role of context, age, and musical experience. Psychology of Music, 26, 7-25.

Lamont, A. (2002). Musical Identities and the School Environment. En R. MacDonald, D. J. Hargreaves, y D. Miell (Eds.), Musical Identities (pp. 41-59). New York: Oxford University Press.

Lamont, A. (2017). Musical Identity, Interest and Involment. En R. MacDonald, D. J. Hargreaves, y D. Miell (Eds.), Handbook of musical identities (pp. 176-196). New York: Oxford University Press.

Lamont, A. y Tarrant, M. (2001). Children's self-steem, identification with participation in music and sport. En Xth European Conference of Developmental Psychology. Uppsaña.

Larson, R. (1995). Secrets in the bedroom: adolescents' private use of media. Journal of Youth and adolescence, 24(5), 535-550.

Larson, R. y Kubey, R. (1983). Television and music: contrasting media in adolescent life. Youth and Society, 15(1), 13-31.

Luyckx, K. y Schwartz, S. J. (2008). Developmental Typologies of Identity Formation and Adjustment in Female Emerging Adults : A Latent Class Growth Analysis Approach, 18(4), 595-619.

MacDonald, R., Hargreaves, D. J. y Miell, D. (2009). Musical identities. En S. Hallam, I. Cross, y M. Thaut (Eds.), The Oxford Handbook of Music Psychology (pp. 462-480). New York: Oxford University Press.

Marcia, J. E. (1966). Development and validation of ego-identity status. Social Psychology, 3(5), 551-558.

Marcia, J. E. (1980). Identity in adolescence. En J. Adelson (Ed.), Handbook of adolescence psychology (pp. 159-187). New York: Wiley.

McCall, G. J. y Simmons, J. L. (1978). Identities and interactions: an examination of human associations in everyday life. New York: Free Press.

McKinlay, A. y McVittie, C. (2017). «Will the Real Slim Shady Please Stan Up?» En R. MacDonald, D. J. Hargreaves, y D. Miell (Eds.), Handbook of musical identities (pp. 137-151). Nueva York: Oxford University Press. 
McPherson, G., Davidson, J. y Faulkner, R. (2012). Music In Our Lives: Rethinking Musical Abilities, Development and Identity. New York: Oxford University Press.

Mead, G. H. (1934). Mind, self and society: from the standpoint of a social behaviourist. Chicago: University of Chicago.

Meeus, W. (2011). The study of adolescent identity formation 2000-2010: A review of longitudinal research. Journal of Research on Adolescence, 21(1), 75-94. https://doi.org/10.1111/j.1532-7795.2010.00716.x

Miranda, D. (2013). The role of music in adolescent development: much more than the same old song. International Journal of Adolescence and Youth, 18(1), 5-22. https://doi.org/10.1080/02673843.2011.650182

Morf, C. C., y Mischel, W. (2012). The Self as a Psycho-Social Dynamic Processing System. Toward a Converging Science of Selfhood. En M. R. Leary y J. P. Tangney (Eds.), The Handbook of Self and Identity (pp. 21-49). Nueva York y Londres: The Guilford Press.

North, A. C. y Hargreaves, D. J. (1999). Music and Adolescent Identity. Music Education Research, 1(1), 75-92. https://doi.org/10.1080/1461380990010107

North, A. C., Hargreaves, D. J. y O'Neill, S. A. (2000). The importance of music to adolescents. British Journal of Educational Psychology, 70(2), 255-272. https://doi.org/10.1348/000709900158083

Organización Mundial de la Salud. (2018). Organización Mundial de la Salud. Recuperado 20 de octubre de 2018, de https://www.who.int/maternal child adolescent/topics/adolescence/dev/es/

Oyserman, D., Elmore, K. y Smith, G. (2012). Self, Self-Concept, and Identity. En M. R. Leary y J. P. Tangney (Eds.), Handbook of Self and Identity (pp. 69-104). Nueva York y Londres: The Guilford Press.

Pardun, C., L'Engle, K. y Brown, J. (2005). Linking Exposure to Outcomes: Early Adolescents' Consumption of Sexual COntent in Six Media. Mass Communication and Society, 8(2), 7591. https://doi.org/10.1207/s15327825mcs0802

Potter, J. y Wetherell, M. (1987). Discourse and social psychology: beyond attitudes and behaviour. Londres: SAGE Publications.

Reisigl, M. (2013). Critical Discourse Analysis. En R. Bayley, R. Cameron, y C. Lucas (Eds.), The Oxford Handbook of Sociolinguistics (pp. 1-24). Nueva York: Oxford University Press.

Roe, K. (1985). Swedish youth and music: listening patterns and motivations. Communication Research, 12(3), 353-362.

Ross, M. (1995). What's wrong with school music? British Journal of Music Education, 12, 185201.

Saarikallio, S. y Erkkilä, J. (2007). The role of music in adolescents' mood regulation. Psychology of Music, 35(1), 88-109. https://doi.org/10.1177/0305735607068889

Schechtman, M. (2011). The Narrative Self. En S. Gallagher (Ed.), The Oxford Handbook of the Self (pp. 1-25). Nueva York: Oxford University Press.

Shaw, S. M., Kleiber, D. A., y Caldwell, L. L. (1995). Leisure and identity formation in male and female adolescents: A preliminary examination. Journal of Leisure Research, 27(3), 245263.

Sneed, J. R., Schwartz, S. J. y Cross, W. E. (2006). A Multicultural Critique of Identity Status Theory 
and Research: A Call for Integration. Identity: an International Journal of Theory and Research, 6(1), 6184. https://doi.org/10.1207/s1532706xid0601

Snell, K. (2009b). Embodied performance in popular music: Considerations for music education through an examination of the Dresden dolls. Journal of Popular Music Studies, 21(1), 5975. https://doi.org/10.1111/j.1533-1598.2009.01169.X

Spychiger, M. B. (2017). From Musical Experience to Musical Identity. En R. MacDonald, D. J. Hargreaves, y D. Miell (Eds.), Handbook of musical identities (pp. 267-287). New York: Oxford University Press.

Stryker, S. (1968). Identity salience and role performance: The relevance of symbolic interaction for family research. Journal of Marriage and the Family, 30, 558-564.

Stryker, S. (2007). Identity Theory and Personality Theory: Mutual Relevance. Journal of Personality, 75(6), 1083-1102.

Tajfel, H. y Turner, J. (1974). Social identity and intergroup behaviour. Social Science Information, 13(2), 65-93.

Tajfel, H. y Turner, J. (1986). The social identity theory of intergroup behaviour. En S. Worchel y W. Austin (Eds.), Psychology of intergroups relations (pp. 7-24). Chicago: Nelson-Hall.

Tarrant, M. y North, A. C. (2000). English and American Adolescents' Reasons for Listening to Music. Psychology of Music, 28, 166-173.

Tarrant, M., North, A. C. y Hargreaves, D. J. (2002). Youth Identity and Music. En R. MacDonald, D. J. Hargreaves, y D. Miell (Eds.), Musical Identities (pp. 134-150). New York: Oxford University Press.

Thomas, K. S. (2016). Music Preferences and the Adolescent Brain: A Review of Literature. Update, 35(1), 47-53. https://doi.org/10.1177/8755123315576534

Trehub, S. E. (2006). Infants as musical connoisseurs. En G. McPherson (Ed.), The Child as Musician. A Handbook of Musical Development, (pp. 33-49).

Trevarthen, C. (2002). Origins of Musical Identity: Evidence from Infancy for Musical Social Awareness. En R. MacDonald, D. J. Hargreaves, y D. Miell (Eds.), Musical Identities (pp. 2138). New York: Oxford University Press.

Trevarthen, C. y Malloch, S. (2017). The Musical Self. En R. MacDonald, D. J. Hargreaves, y D. Miell (Eds.), Handbook of musical identities (pp. 155-175). Nueva York: Oxford University Press.

Ward, L. M., Hansbrough, E. y Walker, E. (2005). Contributions of music video exposure to black adolescents' gender and sexual schemas. Journal of Adolescent Research, 20(2), 143-166. https://doi.org/10.1177/0743558404271135

Westerlund, H., Partti, H. y Karlslen, S. (2017). Identity formation and agency in the diverse music classroom. En Raymons MacDonald, D. J. Hargreaves, y D. Miell (Eds.), Handbook of musical identities (pp. 493-509). New York: Oxford University Press. https://doi.org/10.1093/oxfordhb/9780198722946.013.45

Wetherell, M. (2010). The Field of Identity Studies. En M. Wetherell y C. Mohanty (Eds.), The SAGE Handbook of Identities (pp. 3-26). Londres: SAGE Publications.

Wright, R. (2008). Kicking the habitus: power, culture and pedagogy in the secondary school music curriculum. Music Education Research, 10(3), 389-402. https://doi.org/10.1080/14613800802280134 